\section{Implementación de vías clínicas multidisciplinarias en una institución de salud de alta complejidad}

\author{
PAMELA CHÁVEZ BARRIENTOS ${ }^{1}$, CRISTIAN MENAJOVSKY HAAS ${ }^{2, a}$, \\ LUIS ARRIAGADA BEYZAGA ${ }^{3, b}$
}

\section{Implementation of multidisciplinary clinical pathways in a highly complex health institution}

Clinical pathways or structured multidisciplinary care plans are a tool to standardize clinical practice. The aim of this article is to describe and propose a methodology for the development of clinical pathways in a highly complex health institution. We used the guidelines of the European Quality of Care Pathway, which are based on a 4-stage model: definition and diagnosis, development, implementation and continuity. To make this process operational, we incorporated the project management methodology (PMO). The implemented methodology contemplates the generation of an institutional governance structure responsible for the coordination of the project and the prioritization of the clinical processes to be standardized. The implementation of each clinical pathway was carried out in four steps, namely team generation, development, planning and implementation, and continuous improvement. With this methodology, 23 clinical pathways were developed between 2014 and 2018. Their implementation significantly reduced hospitalization days and their variability, optimized the use of resources and standardized clinical records. The standardization of clinical processes allowed the development of new skills such as the generation of clinical projects, an institutional methodology for managing change and sustainability through cycles of continuous improvement.

(Rev Med Chile 2020; 148: 1331-1338)

Key words: Change Management; Clinical Pathways; Standard of Care; Quality Improvement.

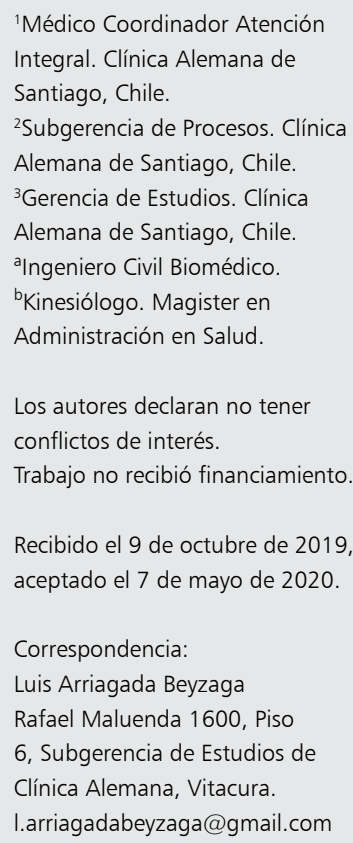

L a personalización e individualización de la práctica clínica sumada a la complejidad de coordinación de la atención médica son unos de los factores que hacen que un mismo problema de salud tenga múltiples maneras de ser tratado, impactando en el proceso de atención y en los recursos utilizados, y con distintos resultados. Una forma de contrarrestar esta situación tan común en salud es por medio de la estandarización de procesos con lo cual se busca disminuir la variabilidad de la práctica clínica a través de varias herramientas que suelen confundirse concep- tualmente, incluso en la literatura: guías clínicas, protocolos y vías clínicas.

Una guía de práctica clínica surge del trabajo de un grupo multidisciplinario de expertos, que recogen la evidencia científica disponible para el manejo de una condición clínica concreta. Por otro lado, los protocolos de trabajo pretenden sintetizar la acción frente a un problema concreto, paso a paso, adaptado al entorno donde se aplica $\mathrm{y}$ a los profesionales que lo utilizan ${ }^{1}$.

La vía clínica (VC) es una intervención para planificar, coordinar y estandarizar el manejo de 
un problema clínico específico en un período definido de su cuidado. Pretende asegurar que el equipo multidisciplinario realice las intervenciones fundamentales, trasladando evidencia científica y guías clínicas a la estructura local, logrando reducir la variabilidad clínica en la práctica $\operatorname{diaria}^{2-4}$. Los objetivos de una VC son: lograr seguridad, efectividad, eficiencia y cuidado centrado en el paciente y su familia; reducir actividades que no aportan valor al proceso; y evitar errores de comunicación en las transiciones ${ }^{5}$.

De acuerdo a la experiencia internacional, las VC promueven y facilitan la práctica del cuidado basado en evidencia mejorando la coordinación del proceso de atención, la comunicación con el paciente, el trabajo en equipo, el seguimiento de los pacientes y la eficiencia ${ }^{4,6}$. En una revisión Cochrane de veinte estudios que comparan el manejo habitual de un problema de salud con la implementación de una VC se observó una reducción de los riesgos de complicaciones, un aumento en la documentación, una reducción de costos y una disminución de los días de estada ${ }^{7}$.

En nuestra institución, la necesidad de avanzar hacia la estandarización de los procesos de atención en salud ha sido una exigencia de los organismos acreditadores de calidad, por lo que se ha definido como una mejora prioritaria. Fue así que se decidió en el año 2014 implementar la metodología de VC como estrategia institucional para generar planes de atención estructurados.

Después de cinco años de iniciado la experiencia en $\mathrm{VC}$ nos proponemos comunicar las lecciones aprendidas, especialmente de las etapas de implementación y mejora continua por su mayor dificultad y menor referencia bibliográfica.

Los objetivos de este trabajo son:

- Describir y analizar el proceso de implementación de VC en nuestra institución, identificando los principales obstáculos y las eventuales soluciones que hemos definido para establecer un sistema de mejora continua.

- Analizar el impacto clínico obtenido con la implementación de algunas VC.

\section{Materiales y Métodos}

\section{Metodología de vías clínica}

Para iniciar el trabajo en VC se utilizaron los lineamientos de la European Quality of Care Pathway ${ }^{8}$, según los cuales la implementación de una
VC se debe basar en un modelo de cuatro etapas:

Etapa 1. Definición y diagnóstico: En esta primera etapa se identifica la brecha entre la situación actual, determinada por indicadores base, y la deseada, identificando objetivos y tiempo de desarrollo de la VC.

Etapa 2. Desarrollo: En este período se desarrolla el proceso asistencial estandarizado mediante un mapa asistencial o árbol de decisión, definiéndose las metas, $y$ analizando el proceso asistencial y su rediseño ${ }^{1,9}$.

Etapa 3. Implementación: Corresponde a la etapa de diseminación por comunicación a todos los involucrados y al monitoreo de indicadores asociado a los objetivos del ciclo ${ }^{10}$.

Etapa 4. Continuidad: En esta etapa el equipo interdisciplinario vigila los indicadores y trabaja los ciclos de mejora continua ${ }^{11}$.

\section{Metodología de Gestión de Proyectos (PMO)}

La metodología de trabajo sugerida por la Oficina de Gestión de Proyectos (PMO, de la sigla en inglés de Project Management Office $)^{12,13}$ plantea que todo proyecto debe ser formalizado mediante una "Ficha de Proyecto" o Project Charter. Esta ficha consiste en un documento simplificado con la información relevante del proyecto: título, sponsors, contexto y alcance, antecedentes, objetivos, hitos y equipo. Paralelamente, la metodología propone un proceso de planificación, el cual consiste en la identificación temprana de todos los involucrados en el proceso asistencial, a quienes se les comparte la "Ficha de Proyecto", la definición de riesgos de implementación y la asignación de responsabilidades.

\section{Resultados}

La implementación de vías clínicas ha sido un proceso de mejora continua en sí mismo. Las primeras seis VC se implementaron bajo la metodología de vías clínicas descrita en la bibliografía y demostró utilidad para grupos pequeños y estructurados en trabajo multidisciplinario. Al momento de intervenir procesos clínicos que incluían a un mayor volumen de profesionales e involucraban a áreas no clínicas, se evidenció la necesidad de gestionar adecuadamente la participación de dichas personas al desarrollo de una VC. Fue así que nos vimos obligados a incorporar la metodología 
de gestión de proyectos al proceso de diseño e implementación. Además hubo dificultades en la comunicación de los procesos clínicos a grupos de gran volumen, por lo que se instauró un plan de difusión institucional al momento de puesta en marcha una nueva VC. Lo anteriormente descrito corresponde a un resumen del camino recorrido durante 5 años que nos ha permitido contar en la actualidad con $23 \mathrm{VC}$ implementadas.

Toda nuestra experiencia en implementación de vías clínicas se materializó en esta metodología de estandarización de procesos clínicos. Esta metodología cuenta con dos grandes etapas de desarrollo complementario: la gobernanza institucional del proyecto global y la metodología de desarrollo e implementación de cada VC.

\section{Gobernanza institucional}

La gobernanza del proyecto de vías clínicas contempla la generación de una estructura de apoyo o soporte institucional y la metodología de priorización de las vías clínicas a trabajar.

La gobernabilidad del Proyecto de Vías Clínicas en la institución requiere como base la implementación de dos grupos de trabajos complementarios:

1) Comité Directivo de Vías Clínicas. Este equipo de trabajo, encargado de alinear el proyecto de vías clínicas a la estrategia institucional, está conformado por un representante directivo de todas las áreas involucradas: Subdirección médica, Jefatura de Calidad, Dirección de Enfermería, Analítica de data y estadísticas, Jefatura de Ficha Clínica Electrónica, Recursos Humanos y Área Comercial.

2) Equipo de Apoyo. Su objetivo es brindar coherencia metodológica al proyecto global. Está liderado por un médico encargado de coordinar el proyecto a nivel institucional y apoyado por representantes del área de Procesos, Analítica de data y estadísticas, Ficha Clínica Electrónica, Recursos Humanos y el área de comunicación institucional hacia Pacientes y Familiares.

Para definir qué procesos clínicos se implementarían se generó una metodología de priorización de VC que contempló la evaluación de las siguientes características: transversalidad respecto a los ámbitos (Ambulatorio, Urgencia, Hospitalizados), multidisciplinaridad, capacidad de trabajo en equipo del equipo clínico objetivo, prevalencia del problema de salud, impacto en la seguridad del paciente, relevancia para un sponsor, nivel de estandarización clínica e impacto en la satisfacción del paciente.

\section{Metodología para cada vía clínica}

La metodología de trabajo de cada VC contempla tres etapas: desarrollo, planificación e implementación, y mejora continua.

\section{Desarrollo}

Cada VC comienza con la conformación del equipo líder de la vía que estará encargado de su desarrollo, implementación y mejora continua. Este equipo está integrado por un médico líder, apoyado estrechamente de otros médicos, enfermería, kinesiología y otras especialidades clínicas según la vía a implementar. La misión del equipo líder es definir los objetivos de la VC, lograr las definiciones y acuerdos de responsabilidad de cada estamento, y hacer seguimiento y mejoras a la VC a lo largo del tiempo.

El equipo ejecutor incluye a todos los profesionales que participan en la asistencia de pacientes de la VC. Deben realizar las actividades estandarizadas, utilizar las herramientas de apoyo a la toma de decisiones y dar cuenta de los problemas que dificulten la acción. La estructura de gobernanza del proyecto de vías clínicas, que incluye la relación entre los distintos grupos involucrados, se resume en la Figura 1.

En reuniones preliminares, el líder del equipo de apoyo junto con el médico líder de la VC abordan el propósito de la estandarización de la práctica clínica, la cohorte de los pacientes a incorporar en la VC, la posibilidad de generar un equipo multidisciplinario y los tiempos estimados para el diseño y la implementación. Posteriormente, se generan las estadísticas que miden el estado actual de la VC definiendo la línea base de trabajo.

La primera etapa de desarrollo de la VC consiste en el mapeo del proceso estandarizado en notación para procesos de negocio (BPMN 2.0). Se define la secuencia de trabajo más apropiada y segura para el paciente, el alcance de la estandarización, las responsabilidades de cada estamento, la posibilidad de mejorar las prácticas existentes con revisión del estado del arte y apego a la cultura institucional y grupal. Se hace énfasis en el mapeo de actividades donde se detectan brechas en la estandarización y apego a la evidencia, y se omiten los pasos altamente estandarizados de antemano. 


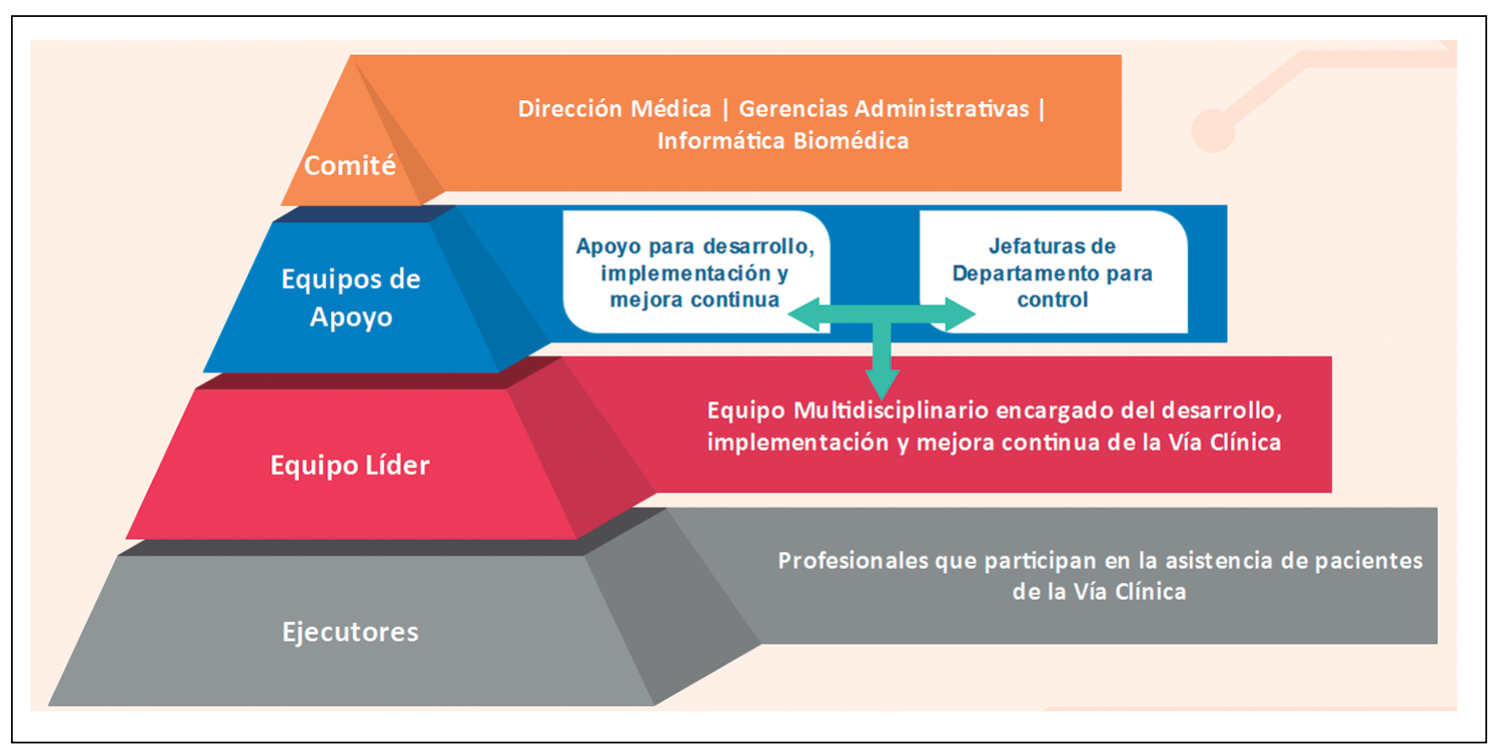

Figura 1. Gobernanza Proyecto Vías Clínicas - Clínica Alemana.

\section{Planificación e implementación}

La planificación se inicia con la elaboración de una Ficha de Proyecto donde se define el nombre oficial de la VC y los interesados del proyecto; se describe el contexto, alcance y objetivos de la $\mathrm{VC}$ en términos genéricos, se identifican los antecedentes estadísticos que justifican la implementación de la VC; y se elaboran entre 3 a 5 objetivos SMART (específicos, medibles, obtenibles, relevantes, acotados en el tiempo) ${ }^{14}$. La Ficha de Proyecto finaliza con el listado de los integrantes del equipo y una referencia a los hitos más importantes del proyecto y sus fechas previstas.

El segundo paso de la planificación consiste en la identificación de los interesados más relevantes para la implementación de la VC. Se incluyen personas y equipos de áreas administrativas y clínicas, y se diseña un plan de comunicación o gestión específico para cada uno. Consistente con el modelo de Kotter de Gestión del Cambio, en esta etapa se hace énfasis en la identificación de líderes informales positivos y negativos que podrían existir en los equipos de trabajo que ejecutarán la VC y constituirán la coalición encargada del cambio. Se recomienda diseñar estrategias para motivar o convertir a estos líderes según el grado de aporte al proyecto ${ }^{15,16}$.

El tercer paso de la planificación consiste en la evaluación de riesgos asociados a la interacción con cada interesado. En caso de existir uno o más riesgos, se objetiva el impacto que tendría cada uno en caso de ocurrir y se diseña un plan de acción orientado a eliminar o mitigar el impacto.

Con los planes de comunicación y mitigación de riesgos, elaboración de documentos específicos de la VC y eventuales planes de gestión del cambio, se elabora una carta GANTT detallada siguiendo un proceso estandarizado: a) listado de todas las tareas; b) ordenamiento lógico secuencial; c) asignación de un responsable; d) estimación de la duración de la tarea; y e) organización lógica de tareas.

Las últimas tareas de la carta Gantt corresponden a las actividades relacionadas con la difusión de la nueva VC en la institución y la capacitación de los equipos clínicos involucrados en el proceso estandarizado.

\section{Mejora continua}

La Mejora Continua de las vías clínicas está basada en constantes ciclos PDSA (planificar, hacer, estudiar y actuar). Esta metodología consiste en realizar ciclos de iteración en intervalos de tiempo pequeños en donde se pueda identificar una oportunidad de mejora, implementarla, estudiar el impacto en indicadores y agregarla al flujo estándar de la forma de trabajar ${ }^{17}$. En caso que uno o más indicadores estén bajo la meta, se investiga 
Vías clínicas en institución de salud de alta complejidad - P. Chávez Barrientos et al

la causa raíz del problema para así implementar una nueva estrategia de mejora.

\section{Impacto evidenciado}

Entre los años 2014 y 2019 se han logrado implementar $23 \mathrm{VC}$, alcanzado en algunas las mejoras esperadas y en otras no se han evidenciado cambios relevantes (Tabla 1). Considerando como criterio la mejora sostenida en dos o más indicadores, se evidencia que $12 \mathrm{VC}$ presentan mejoras, 8 no presentan cambios relevantes y 3 no cuentan con información suficiente de seguimiento.

El impacto más directo contempla los indicadores de procesos de cada vía en donde se ha logrado, por ejemplo, la disminución en los tiempos de estada y su variabilidad (VC fractura de cadera), la optimización del uso de recursos (disminución del uso de radiografía de columna en VC de dolor lumbar), la estandarización en el registro de los parámetros clínicos (registro de frecuencia respiratoria en VC de bronquiolitis), reforzamiento del cumplimiento de normativas de alcance nacional (notificación de las Garantías Explícitas en Salud oral en VC de embarazo), mejoras en la oportunidad diagnóstica (disminución en los tiempos de espera en las biopsias en VC de nódulo mamario) y estandarización en la categorización de la severidad de los diagnósticos, como sucedió en las vías clínicas de dolor lumbar y bronquiolitis. Un ejemplo de mejora significativa corresponde

Tabla 1. Vías clínicas implementadas, año de implementación y resultado

\begin{tabular}{|c|c|c|}
\hline Vía Clínica & Año de Implementación & Resultado \\
\hline Fractura de cadera en adultos mayores (urgencia) & 2014 & $\uparrow$ \\
\hline Fractura de cadera en adultos mayores (hospitalizado) & 2015 & $\uparrow$ \\
\hline Crisis asmática (urgencia) & 2017 & $\uparrow$ \\
\hline Bronquiolitis (urgencia) & 2017 & $\uparrow$ \\
\hline Rodilla traumática aguda (urgencia) & 2017 & $\rightarrow$ \\
\hline Rodilla traumática aguda (hospitalizada) & 2017 & $\rightarrow$ \\
\hline Embarazo normal & 2017 & $\rightarrow$ \\
\hline Nódulo tiroídeo & 2017 & $\uparrow$ \\
\hline Colectomía laparoscópica electiva & 2017 & $\uparrow$ \\
\hline Nódulo mamario sospechoso & 2017 & $\uparrow$ \\
\hline Manejo del dolor postquirúrgico (bomba PCA endovenosa) & 2017 & $\uparrow$ \\
\hline Manejo del dolor postquirúrgico (catéter epidural) & 2017 & $\rightarrow$ \\
\hline Manejo del dolor postquirúrgico (catéter perineural) & 2017 & $\rightarrow$ \\
\hline Bronquiolitis (hospitalizado) & 2018 & $\rightarrow$ \\
\hline Cirugía cardiaca & 2018 & $\rightarrow$ \\
\hline Infarto agudo al miocardio & 2018 & $\rightarrow$ \\
\hline Trombosis venosa profunda (urgencia) & 2018 & $\uparrow$ \\
\hline Trombosis venosa profunda (hospitalizado) & 2018 & $\uparrow$ \\
\hline Delirium (prevención) & 2018 & $\uparrow$ \\
\hline Delirium (tratamiento) & 2018 & $\uparrow$ \\
\hline Linfoma de Hodgkin & 2018 & NA \\
\hline Accidente cerebrovascular isquémico & 2018 & NA \\
\hline Sospecha de cáncer de próstata & 2018 & NA \\
\hline
\end{tabular}

$\uparrow$ Mejoran dos o más indicadores. $\rightarrow$ Mejora solo uno o ningún indicador. NA: Registro insuficiente para evaluar impacto. 


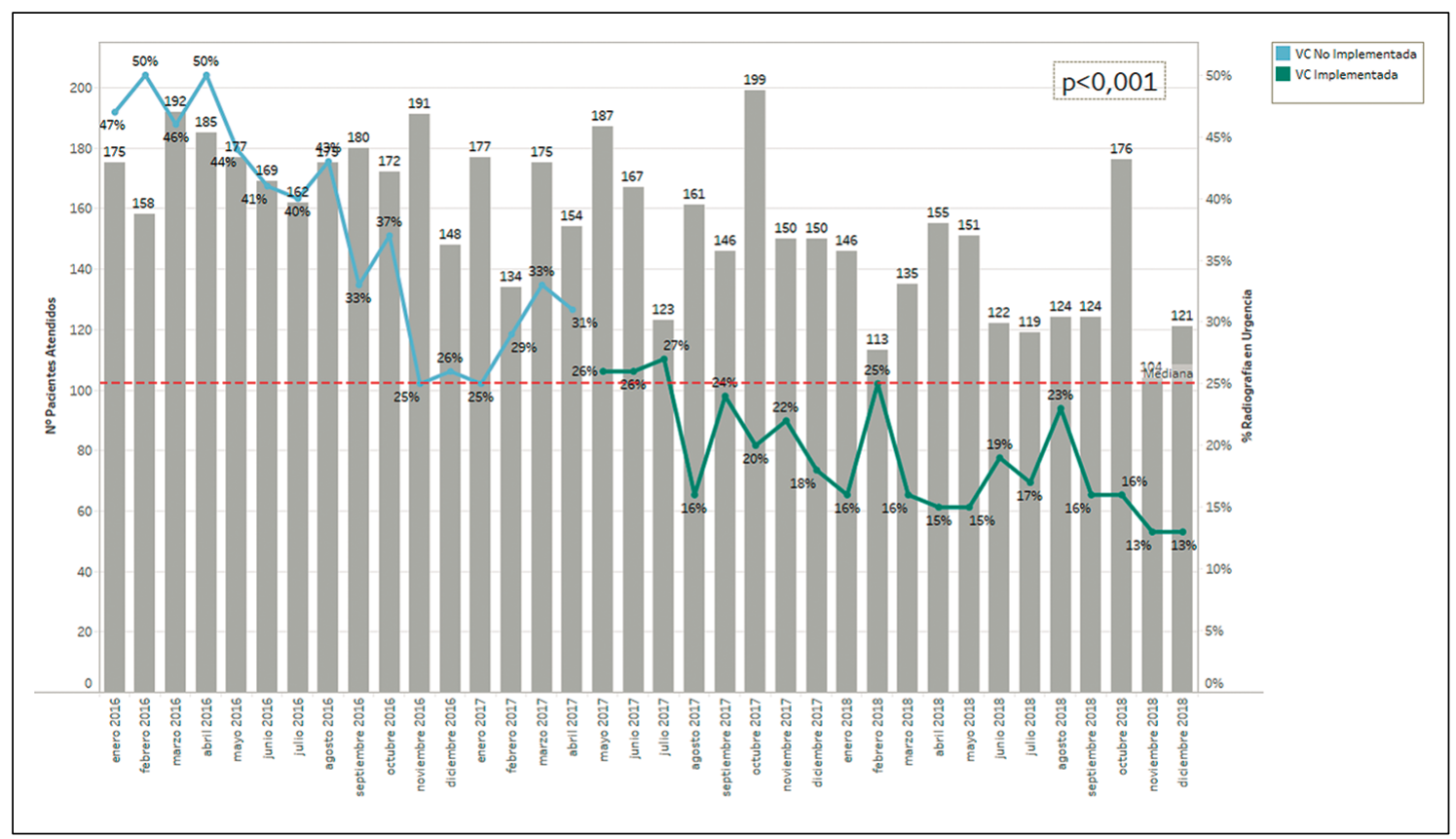

Figura 2. Gráfico indicador solicitud de radiografía de columna - vía clínica dolor lumbar.

a la disminución de días de estada en la VC de fractura de cadera y la disminución del uso de radiografías de columna en la $\mathrm{VC}$ de manejo de dolor lumbar en urgencia (Figura 2), en donde se evidencia que posterior a la implementación 6 o más registros se encuentren bajo la mediana de todo el período de análisis ${ }^{18}$.

Sin perjuicio de los éxitos mencionados, algunos resultados no cumplieron las expectativas del proyecto, como por ejemplo, en el manejo multidisciplinario del embarazo y en la disminución de la estancia hospitalaria de pacientes con colectomía laparoscópica electiva, entre otros.

\section{Discusión}

Las vías clínicas como herramienta de estandarización de prácticas clínicas cuentan con experiencia comunicada por hospitales en USA y Europa hace 20 años $^{19}$. Nuestra institución ha asumido un compromiso en acercar las buenas prácticas con evidencia científica a los pacientes a través de la instalación de VC teniendo como referencia las experiencias publicadas en la litera- tura y, en especial, a The Children's Hospital of Philadelphia ${ }^{20}$.

En términos generales, la experiencia de implementar 23 vías clínicas en nuestra institución ha demostrado que la generación de una estructura posibilita la instalación de una conducta hacia la estandarización; es decir, a partir de la generación de una organización y el uso de una metodología con soporte en el Registro Clínico Electrónico y el desarrollo de estrategias de apoyo a la toma de decisiones, es posible generar cambios en los modelos mentales de los profesionales y autoridades involucradas, lo que su vez fortalece progresivamente dicha estructura en un círculo virtuoso.

Esta experiencia ha demostrado que a través del examen de la estructura interna y del análisis periódico de resultados de procesos (mejora continua) es factible detectar brechas y comprender las resistencias y facilitadores a la incorporación de estándares relevantes para la práctica clínica a través de metodologías de gestión del cambio. Es crítico lograr el involucramiento vertical (jerárquico) y horizontal (interdisciplinario), tanto de estamentos clínicos como administrativos, para el éxito de la tarea desde sus primeras etapas y 
especialmente en el proceso de mantención. Para ello consideramos que el rol de un Líder de equipo es fundamental, por lo que es necesario definir un perfil deseable para esta responsabilidad en cada institución.

Las principales dificultades a las cuales nos enfrentamos en el desarrollo, implementación o mejora continua de una VC están relacionadas con una falta de cultura de trabajo en equipo donde los grupos interdisciplinarios, especialmente médicos, no logran las sinergias que se requieren para actuar de modo colaborativo, por lo cual consideramos fundamental hacer una evaluación previa de las capacidades de los equipos. Así también nos encontramos con la resistencia al cambio por parte de los profesionales, no acostumbrados a la revisión de sus procesos asistenciales, lo cual es percibido como una evaluación personal más que una oportunidad de mejora al proceso para asegurar resultados a los pacientes. La falta de consideración de estas dificultades implica un mayor tiempo de desarrollo e implementación de cada VC por lo que deben ser detectadas y trabajadas oportunamente para neutralizar su impacto en el proyecto.

Entre las iniciativas a trabajar para mejorar el desempeño de las vías clínicas se encuentran la definición del perfil de líder que permita sostener la $\mathrm{VC}$ en el tiempo con incentivos adecuados para los clínicos, el desarrollo de una metodología para la autogestión de los equipos ejecutores en la etapa de mejora continua, la optimización del proceso de análisis de datos y la obtención de indicadores, el desarrollo de herramientas de apoyo a la toma de decisiones, la incorporación de la visión del paciente y la adaptación de esta metodología para su aplicación en el desarrollo e implementación de protocolos clínicos.

Esta propuesta de implementación está adaptada a una institución que está iniciando un cambio cultural hacia la estandarización de los procesos clínicos. Existe evidencia de otras metodologías de implementación de $\mathrm{VC}$ que muestran una rápida diseminación y un alto nivel de usabilidad en una red de prestadores ${ }^{21}$. Sería relevante en el futuro comparar los resultados de estandarización entre estas dos metodologías.

Para una adecuada implementación de vías clínicas en una institución de salud creemos que es prioritario adaptar la metodología a la realidad local ya que la intensidad de los cambios a realizar va a depender de la cultura organizacional de la institución. Según nuestra experiencia, recomendamos contar con un líder institucional a cargo del proyecto global y comenzar con una VC piloto, para posteriormente establecer la estructura institucional de soporte del proyecto. A medida que la metodología de vías clínicas se va interiorizando en la organización creemos que es relevante capacitar a los equipos en gestión clínica, gestión del cambio y en habilidades de liderazgo, además de potenciarlos en herramientas de manejo y análisis de datos (Excel y business intelligence), y comunicación efectiva para un óptimo feedback a los equipos ejecutores.

\section{Referencias}

1. Carrasco G, Ferrer J. Las vías clínicas basadas en la evidencia como estrategia para la mejora de la calidad: metodología, ventajas y limitaciones. Rev Calidad Asistencial 2001; 16: 199-207.

2. Kinsman L, Rotter T, James E, Snow P, Willis J. What is a clinical pathway? Development of a definition to inform the debate. BMC Medicine 2010; 8: 31.

3. Panella M, Marchisio S, Di S. Reducing clinical variations with clinical pathways: do pathways work? Int J Qual Health Care 2003; 15 (6): 509-21.

4. Vanhaecht K, Bollmann M, Bower K, Gallagher C, Gardini A, Guezo J, et al. Prevalence and use of clinical pathways in 23 countries - an international survey by the European pathway association E-P-A.org. J Intgr Care Pathways 2006; 10: 28-34.

5. Premier, Inc., and Institute for Healthcare Improvement. Integrated Care Pathway for Total Joint Arthroplasty. Charlotte, NC: Premier, Inc. and Cambridge, MA: Institute for Healthcare Improvement; 2013.

6. Deneckere S, Euwema M, Lodewijckx C, Panella M, Mutsvari T, Sermeus W, et al. Better interprofes- sional teamwork, higher level of organized care, and lower risk of burnout in acute health care teams using care pathways: a cluster randomized controlled trial. Med Care 2013; 51 (1): 99-107.

7. Rotter T, Kinsman L, James E, Machotta A, Gothe $\mathrm{H}$, Willis J, et al. Clinical pathways: effects on professional practice, patient outcomes, length of stay and hospital costs. Cochrane Database Syst Rev 2010; 3: CD006632.

8. Deneckere S, Euwema M, Lodewijckx C, Panella M, Sermeus W, Vanhaecht K. The European quality of care pathways (EQCP) study on the impact of care pathways on 
interprofessional teamwork in an acute hospital setting: study protocol: for a cluster randomised controlled trial and evaluation of implementation processes. Implement Sci 2012; 7: 47.

9. Davis N, Integrated Care Pathway. A guide to good practice. National Leadership an Innovation Agency for Heathcare. 2005 http://www.wales.nhs.uk/sitesplus/ documents/829/integratedcarepathways.pdf

10. Kennedy P, Leathley C, Hughes C. Clinica practice variation. MJA 2010; 193: S97-9.

11. García C, Diez S, Chamorro L, Navas A, Vidal F. Vías Clínicas. Hospital Universitario La Paz. Madrid 2010, http://www.chospab.es/calidad/archivos/Vias/elaboracionviasclinicas.pdf.

12. Project Management Institute. (2004). A guide to the project management body of knowledge (PMBOK guide). Newtown Square, Pa: Project Management Institute.

13. Menajovsky C, Copetta C, Prieto C. Documento Interno N. 5009 Clínica Alemana, http://viasclinicas.alemana.cl/ docs_generales/paper_metodologia_v4.pdf

14. MacLeod L. Making SMART goals smarter. Physician executive 2012; 38 (2): 68-72.

15. Kotter JP. Leading Change. Boston: Harvard University Press, 1996.

16. Narine and Persaud, Gaining and maintaining commitment to large-scale change in healthcare organizations. Health Services management centre 2003.

17. NHS, Plan, Do, Study, Act (PDSA) cycles and the model for improvement. https://improvement.nhs.uk/documents/2142/plan-do-study-act.pdf

18. Francis R, Adam S, Harel Z, Weizman A, Thomas A, Bell C, et al. Clin J Am Soc Nephrol 2016; 11: 908-14.

19. Campbell H, Hotchkiss R, Bradshaw N, Porteous M. Integrated care pathways. BMJ 1998; 316: 133-7.

20. Lavelle J, Schast A, Keren R. Standardizing Care Processes and Improving Quality Using Pathways and Continuous Quality Improvement. Current Treatment Options in Pediatrics December 2015, Volume 1, Issue 4, pp 347-58.

21. Flores EJ, Mull N, Lavenberg J, Mitchell M, Leas B, Williams A, et al. Using a 10-step framework to support the implementation of an evidence-based clinical pathways programme. BMJ Qual Saf 2018; 0: 1-10. 